1

2

3

\title{
Subject-specific musculoskeletal loading of the tibia: Computational load estimation
}

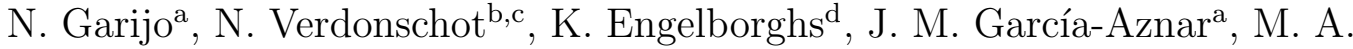 \\ Pérez $^{\mathrm{a}}$ \\ ${ }^{a}$ Multiscale in Mechanical and Biological Engineering (M2BE) \\ Aragón Institute of Engineering Research (I3A). University of Zaragoza \\ ${ }^{b}$ Laboratory for Biomechanical Engineering \\ University of Twente, Enschede, The Netherlands \\ ${ }^{c}$ Radboud University Medical Center, Radboud Institute for Health Sciences, \\ Orthopaedic Research Laboratory, Nijmegen, Netherlands \\ ${ }^{d}$ Biomedical Engineering Department, Materialise NV, Leuven, Belgium
}

\begin{abstract}
The systematic development of subject-specific computer models for the analysis of personalized treatments is currently a reality. In fact, many advances have recently been developed for creating virtual finite element-based models. These models accurately recreate subject-specific geometries and material properties from recent techniques based on quantitative image analysis. However, to determine the subject-specific forces, we need a full gait analysis, typically in combination with an inverse dynamics simulation study. In this work, we aim to determine the subject-specific forces from the computer tomography images used to evaluate bone density. In fact, we propose a methodology that combines these images with bone remodelling simulations and artificial neural networks. To test the capability of this novel technique, we quantify the personalized forces for five subject-specific tibias using our
\end{abstract}

Email address: angeles@unizar.es (M. A. Pérez) 
technique and a gait analysis. We compare both results, finding that similar vertical loads are estimated by both methods and that the dominant part of the load can be reliably computed. Therefore, we can conclude that the numerical-based technique proposed in this work has great potential for estimating the main forces that define the mechanical behaviour of subjectspecific bone.

Keywords: Artificial neural network, subject-specific, bone density, musculoskeletal model, bone remodelling problem/inverse bone remodelling model

\section{Introduction}

Subject-specific models are becoming increasingly important because of the clinical demands of patient-centered treatments. Advancements in different current technologies including computed tomography (CT), magnetic resonance imaging $(\mathrm{MRI})$, and gait analysis have enabled the creation of more realistic subject-specific computational-based bone models (Lekadir et al., 2015). Subject-specific modelling often starts with previously acquired images that can provide information regarding the geometry and density distribution of the individual bone properties. However, direct subject-specific estimation of bone loads through in vivo imaging remains challenging (Zadpoor and Weinans, 2015).

The combination of subject-specific joint and muscle force-based models with consistent bone geometry into finite element-based (FE) models could be a very important advancement for creating subject-specific models that allow for predictive analyses of personalized treatments. Vahdati 
et al. (2014) combined gait analysis and a subject-specific musculoskeletal model with subject-specific bone geometry in a computational bone remodelling methodology for predicting bone density distribution. The results confirmed that the predicted bone density distribution in the proximal femur was drastically influenced by the inclusion of subject-specific loading conditions. González-Carbonell et al. (2015) used the subject-specific geometry and material properties to study the tibial torsion using CT. Additionally, Carey et al. (2014) created subject-specific FE models of the tibiofemoral joint using dynamic stereo-radiography data and kinematic analysis. Although these aforementioned models provided full information on bone mechanical properties, several difficulties could arise before their methods can be applied clinically due to the amount of initial information required.

Moreover, musculoskeletal models have been useful tools for virtual orthopedic surgery. Inverse dynamics techniques were used in gait analysis to calculate the net joint torques that the musculoskeletal system produces during human locomotion (Liu et al., 2009; Favre et al., 2012). In recent decades, multiple methods have been developed to improve the performance of subject-specific models (Fluit et al., 2012, 2014). Carbone et al. (2012) showed errors in the estimated position of muscle attachment sites that affected muscle force predictions. Subsequently, Carbone et al. (2015) combined morphing of bone surfaces with muscle volumes and functional optimization of muscle-tendon architecture to create a musculoskeletal geometry dataset. This part was linked with muscle-tendon attachment sites and lines-of-action (Pellikaan et al., 2014), or muscle volumes (Carbone et al., 2012), showing that subject-specific models resulted in more reliable out- 
comes, whereas conventional anthropometric scaling laws were inadequate and provided less realistic muscle activity predictions. These complex models were, however, troublesome with regard to their immediate application to patients.

For most of these methodologies, it is not easy to prove the clinical benefits due to the complex process involved and their large computational cost. In addition, estimating musculoskeletal loads requires information about the movements of the individual patient. Note that it is very difficult to measure loads in vivo using non-invasive procedures.

Several studies have attempted to estimate loads by solving the inverse bone remodelling problem using different numerical approaches. In fact, Fischer et al. (1995) developed an optimization procedure that adjusted the magnitude of each basic load in 2D to achieve the desired bone density. Bona et al. (2006) proposed a contact algorithm for density-based load estimation and used a method to distinguish between different modes of locomotion of animals. More recently, Christen et al. (2012) developed a bone loading estimation algorithm to predict loading conditions through calculating the loading history that produces the most uniform strain energy density on the bone tissue. Campoli et al. (2012) were the first to use the artificial neural network (ANN) approach to predict femur loads from the bone density distribution. These authors combined a wavelet decomposition technique with an ANN to estimate the loading parameters of the femur. Zadpoor et al. (2013) also used ANN to predict tissue adaptation loads from a given density distribution of trabecular bone in a 2D example of the femur. Garijo et al. (2014b) presented a numerical methodology in which the specific load 
that the bone was actually supporting was predicted through different mathematical techniques by utilizing the bone density distribution obtained from bone remodeling simulations. They used a single femur, and they theoretically predicted the loading conditions that induced a virtual bone density distribution with good accuracy using ANN.

However, in this work we present a general computational-based methodology to determine the forces that a subject-specific tibia is supporting from the CT images of this specific patient. For this purpose, we used five subjectspecific tibias, from which knowing their bone geometry and density distribution, we will predict their specific loading conditions. Finally, to quantitatively validate the predictive capacity of this novel methodology, we will compare these forces with those obtained for each subject from an individualbased gait analysis and subsequent musculoskeletal force prediction.

\section{Materials and Methods}

To determine subject-specific loads acting on the tibia, a computationalbased approach was developed, which combined different numerical tools widely used in bone image analysis and bone mechanics. Thus, we first describe this computational approach to determine the subject-specific forces. Next, we present the method used to validate this novel methodology. Finally, we present the final subject-specific cases that were studied.

\subsection{Computational-based methodology for estimating subject-specific loads.}

[Fig. 1 about here.]

To apply this methodology (see Fig. 1), we required the subject-specific bone geometry and its bone apparent density, which can be obtained from 
individual CT data (Hounsfield Units - HU) (Section 2.3.2) through current standard image analysis (Bitsakos et al., 2005). Therefore, from this analysis, we were able to construct a subject-specific FE model that replicated the main characteristics of the bone: its geometry and heterogeneous material properties (Fig.1-left). This FE model was used for intensive bone remodelling simulations (Doblaré and García, 2001) (see Appendix A) with multiple different load cases that come from inter- and intra-subject variability (Motion data - Fig.1-right). The knee joint force was assumed to define the bone density distribution (Fx, Fy and Fz). From the multiple bone remodelling simulations, we obtained different apparent density patterns for each load condition. Then, we selected the apparent density and volume at different regions of interest (ROIs) (Appendix B) defined for the tibia in all the analyses. These data (ROIs from remodelling) and their corresponding loads (from the motion database - Fx, Fy and Fz) were used to train the ANN (see Appendix C). Through multiple iterations, the ANN was trained, obtaining a correlation between the ROIs and forces (Fig. 1).

After training the ANN (Fig. 1- bottom left), we introduced the density and volume values at the ROIs (Appendix B) from the subject-specific CT data (HU) as input data in the ANN. Subsequently, the ANN will predict the subject-specific forces (output value - Fx, Fy and Fz) (Fig. 1).

This process was repeated for the five considered tibias. Therefore, we developed five different subject-specific ANNs, one for each subject.

\subsection{Validation of the model through gait analysis}

To validate the performance of the ANN for tibia loading prediction in each subject-specific tibia, we compared previous ANN-based predicted loads 
(section 2.1) with the loads obtained from the subject-specific musculoskeletal analysis (see Fig. 2) and the 3D gait measurements (Fluit et al., 2014). 3D gait analyses, regarding kinematics and ground reaction forces of the subjects, were performed using the Twente Lower Extremity Model (TLEM 2.0) and AnyBody Modeling System ver. 6.0.2 (Anybody Technology A/S, Aalborg, Denmark). TLEM 2.0 is a new comprehensive data set of the musculoskeletal geometry of the lower extremity, and it is based on medical imaging data (Carbone et al., 2015).

[Fig. 2 about here.]

\subsection{Subject-specific data and FE models}

Data of five healthy subjects were utilized. The subjects had no history of major injury and had not undergone orthopedic surgery on the lower

limbs. We specifically selected the subjects to include a wide variety of subjects in terms of age (23-61 years), gender (two men and three women) and weight (58 kg to $90.4 \mathrm{~kg}$ ) (Table 1). The procedures developed in this study were approved by the ethics committee of the region of Arnhem-Nijmegen (Netherlands). A written informed consent was obtained from each subject.

[Table 1 about here.]

\subsubsection{Subject-specific geometry}

To model the subject-specific geometry, full computational tomography (CT) scans of each subjects' left leg region were obtained (Kolk et al., 2014). A CT scan (SIEMENS/Biograph40) of each subject's legs was obtained with the following specifications: pixel size $=0.977 \mathrm{~mm}$, slice thickness $=3.000 \mathrm{~mm}$, 
pixel resolution $=512 \times 512$ pixels, field of view $(\mathrm{FOV})=500.0 \mathrm{~cm}$, and excitation voltage $=120 \mathrm{kV}$. The $\mathrm{CT}$ images were imported to Mimics ${ }^{\circledR} 17.0$ (Materialise, Leuven, Belgium). We performed a semiautomatic segmentation of the left tibia and fibula and their corresponding 3D geometrical reconstruction of each bone. Subsequently, a joint area was created between the tibia and fibula that simulated the cartilage between both bones. The FE meshes were generated by 3-matic ${ }^{\circledR}$ (Materialise, Leuven, Belgium) using a semi-automatic meshing procedure.

Every tibia mesh was constructed using linear tetrahedral elements with an element size of approximately $3 \mathrm{~mm}$ (see Fig. 3a). The element size was inside the asymptotic region of convergence and represented a good trade-off between numerical accuracy and computational cost for all cases.

Subsequently, we defined the anatomical landmarks and joint centres for every subject and the local reference frame for load application. The load characteristics (Section 2.3.3) were expressed relative to the local reference frame (see Appendix D) and were applied through rigid beams that connected the rotation centre of the tibia-fibula joint $(\mathrm{RJ})$ with a surface over the proximal tibial condyles. Bilinear quadrilateral elements were created in the surface of the proximal condyles to apply loading conditions uniformly distributed in the knee joint (Fig. 3a). This process was repeated for each subject.

Therefore, the beams and the layer of elements over the proximal tibia condyles were assumed to be rigid. For the cartilage elements (joint between tibia and fibula), Young's modulus and Poisson's ratio were assumed to be 10.0 MPa and 0.45, respectively (Jin and Lewis, 2004), neglecting its biphasic 


\section{behavior.}

For the bone remodelling simulations (Sections 2.1- Appendix A), each tibia was initially assigned a uniform bone density of $0.5 \mathrm{~g} / \mathrm{cm}^{3}$ and was fully constrained distally throughout the entire analysis (Fig. 3). Changes in the bone density distribution were computed until convergence. More details can be found in Doblaré and García (2001) (see Appendix A).

[Fig. 3 about here.]

\subsubsection{Subject-specific density from $H U$}

We need the subject-specific bone density from every patient to be the input for every subject-specific ANN. Therefore, for computing the density from the HU values (Figs. 1 and 2), the FE meshes were imported into Mimics ${ }^{\circledR} 17.0$ again, and different material properties were assigned by relating the bone mineral density with the HU. In the literature, different relationships between bone density and HU can be found for different bone types, primarily for the lower extremities (Peng et al., 2006). In the present study, we used a linear relationship, as proposed by Bitsakos et al. (2005).

From each subject's CT data (see Table 1), the HU maximum (HUmax) and minimum (HUmin) values were obtained and were correlated with bone density values of $1.92 \mathrm{gr} / \mathrm{cm}^{3}$ and $0.5 \mathrm{gr} / \mathrm{cm}^{3}$, respectively. These values were introduced into eq. 1 , and the bone density value at every point was computed as follows:

$$
\rho_{i}=0.5+\frac{1.92-0.5}{H U_{\max }-H U_{\min }}\left(H U_{i}-H U_{\min }\right)
$$

These density values were used as inputs for the ANN-based simulations to predict the subject-specific tibia loading conditions (Figs. 1 and 2). 


\subsubsection{Subject-specific musculoskeletal loads}

Each subject performed different gait trials, and their data were calculated using TLEM 2.0 (Fluit et al., 2014; Carbone et al., 2015). Subjectspecific musculoskeletal loads were used to define the load patterns that are applied to the tibia. We assumed that the knee joint force is the one that predominantly defines the bone density distribution of the tibia. It was expressed in the local reference frame of the tibia and fibula (Appendix D). Using the subject-specific musculoskeletal load data, we evaluated the knee joint forces in the $\mathrm{X}, \mathrm{Y}$ and $\mathrm{Z}$ axes for all the subjects involved, thereby computing the maximum force values (see Fig. 4). Table 2 shows the maximum force in each direction for each subject.

\subsubsection{Bone remodelling loading conditions}

For the bone remodelling simulations, we further assumed that the maximum forces $\mathrm{X}, \mathrm{Y}$ and $\mathrm{Z}$ observed during walking were independently and consecutively applied to simulate tibia loading. These three consecutive loading cases were applied (Fy, Fx and Fz) acting in blocks of 10000 direct cycles but with different frequencies for each one (6000, 2000 and 2000, respectively) (Doblaré and García, 2001) (Appendix A). We assumed that the dominant load (Fy - force in the vertical direction of the tibia) has a high frequency of occurrence with respect to Fx and Fz. These loads were applied through the position of the centre of the knee joint (Appendix D).

[Fig. 4 about here.]

Different loading conditions that take inter-individual variations into account were simulated to obtain their corresponding bone density distributions 
for training of the ANN. We computed every mean load value as the mean between the maximum and minimum load of every subject (Table 2). Then, we calculated the difference between the mean and every force component (maximum difference is 24\%). Therefore, we selected a slightly wide range of variation (35\%). We considered that this range of variation was representative of the majority of our population.

Finally, nine variations of each force value (Fx, Fy and Fz) were considered within the $35 \%$ range of variation. When one force value was varied, the rest of the forces remained constant. To summarize, 729 (9x9x9) combinations of loading parameters were simulated for each subject. Therefore, a total of 729 bone remodelling problems were initially solved for each subject (see Fig. 1) to train each subject-specific ANN. The training of each ANN was performed using the 12 ROI values of each of the 729 bone remodelling simulations as inputs and their corresponding loads (considered as outputs of the ANN) (see Appendix C).

[Table 2 about here.]

All the FE simulations were performed in Abaqus v6.14. (Dassault Systèmes Simulia Corp., Suresnes, France) and run in a computational cluster of 224 cores and 576 GB of RAM.

\subsection{Performance of the ANN-based simulations}

To predict the accuracy of this technique, the absolute relative error (RE) and the correlation coefficient (RSQ) were computed using the following expressions: 


$$
\begin{gathered}
R E=\operatorname{abs}\left(\frac{\hat{\theta}-\theta}{\theta}\right) \\
R S Q=\frac{\sigma_{\hat{\theta} \theta}}{\sigma_{\hat{\theta}} \sigma_{\theta}}
\end{gathered}
$$

where $\hat{\theta}$ is the predicted data, $\theta$ is the real data (reference values), $\sigma_{\hat{\theta} \theta}$ is the covariance, and $\sigma_{\hat{\theta}}$ and $\sigma_{\theta}$ are the standard deviations of all the involved data.

The relative error results were calculated based on the training (90\% of the 729 bone remodelling simulations) and the testing sets (10\% of the 729 bone remodelling simulations) of the mean 10 -fold cross validation (see Appendix C). The training data allow generation of the model, and thus, the corresponding error value set indicates whether the model has been well trained, and the test data are used to validate the model. The corresponding error indicates whether the model is appropriate for solving the problem.

The ANN performance tests were implemented in Matlab R2013a.

\section{Results}

\subsection{Quantitative performance of the ANN-based simulations}

The performance of each subject-specific ANN needs to be checked to determine its suitability for the present problem. Table 3 shows the mean error of the results obtained for each case. In some cases, the RE during training was higher than during testing, but the RE computed during training and testing were lower than 1\%. Moreover, the RSQ was very close to 1 . This result indicates that each subject-specific ANN was well trained and that the force estimation can be considered accurate. 
[Table 3 about here.]

\subsection{Quantitative comparison of personalized forces between gait analysis pre- dictions and $A N N$-based analysis}

To validate our methodology, we compared the forces predicted by each subject-specific ANN (Section 2.1) with the forces obtained by each subjectspecific gait analysis (Table 2 and Section 2.2). We computed the relative error through the comparison between both forces.

[Table 4 about here.]

As shown in Table 4, Force $\mathrm{Y}$ was accurately predicted in all cases. The values of this force were considerably higher than the others (Fx and Fz) in all the subjects (see Table 2). Therefore, because the forces in Y were higher, it was more difficult to estimate the forces in the $\mathrm{X}$ and $\mathrm{Z}$ directions, thus causing larger errors in those directions. However, when we computed the force error through its total force, the error was less than $6 \%$, which clearly indicated a good prediction.

To demonstrate that the force predictions were truly subject specific, we computed the relative error when subject-specific inputs were interchanged between subjects and introduced in another subject-specific ANN (Table 5). For example, we introduced the input data of subject 1 into ANN-subject 5. Then, the predicted total forces were compared with the gait analysis of subject 1 .

In all cases, except for subject 1 , the error was minimum if we compared the predicted and gait analysis forces when the inputs were introduced in their corresponding subject-specific ANN. 
Only in certain cases was the prediction obtained by interchanging the subject-specific ANN accurate. For example, when introducing subject 3 input data (HU) into subject-specific ANN 4, the error between the forces is $9.84 \%$. In the opposite case, the error was $0.61 \%$ (Table 5 ). In fact, the force errors of subjects 3 and 4 were the same order of magnitude when their input data were introduced into another subject-specific ANN (Table 5).

[Table 5 about here.]

\section{Discussion}

There is an increasing need to create a systematic methodology to predict personalized musculoskeletal loads for subject-specific models. This information will be of considerable value in subject-specific musculoskeletal treatments (such as orthopedic surgery), particularly if it can be easily obtained in a short period of time. Currently, the subject-specific forces are obtained through the development of corresponding musculoskeletal models. Therefore, a rather extensive subject-specific gait analysis is required. These simulations may be accurate and complete, providing information of all muscles (position, load values, and so forth) during the walking cycle (Fluit et al., $2012,2014)$; however, it is occasionally not easy to apply in a clinical setting. In addition, in certain situations (such as in tumour cases), the subject is not allowed to participate in an extensive gait analysis study because there is a risk of pathological bone fracture or other complications.

Garijo et al. (2014b) proposed a novel computational-based technique that combined ANN and FE method to estimate forces in bone. In that work, the validation was purely theoretical and they were not able to test 
it with real subject-specific data. In the present work, we test this previous computational-based technique in order to analyze the bone density distribution associated to five different specific patients. In fact, we present a novel methodology capable of predicting the forces acting on the tibia that each specific subject is experiencing based on their CT images. It is possible to obtain the specific geometry and the density for our methodology from a simple CT scan, and it is not necessary to add any special requirements for the subject. Therefore, this methodology can easily be applied in clinical settings. In the current study, our methodology has been tested in five subjects, obtaining promising results. The comparison with gait analysis demonstrates the good accuracy of our methodology. Errors relative to the total force are less than $6 \%$. Nevertheless, we have to keep in mind that our numerical approach is based on some simplifications. The most relevant simplification is that we only considered the knee contact force acting on the tibia. Actually, we assume that the knee joint force is the driving force controlling the bone density distribution, neglecting the effect of other involved muscle forces. This is the major limitation of our study, and this fact undermines the predictive capacity of the model as the physiologically-relevant mechanical loads experienced by the tibia are much more complex than the loading conditions simulated in our current study. In a future work, this limitation should be reduced performing a deep analysis of the tibia muscle loads.

From the musculoskeletal model, we observe that the values of the Fx and $\mathrm{Fz}$ forces (horizontal forces) are not very high and that their variability during the walking cycle is extremely small $(\mathrm{Fx}=137.3 \pm 15 \%$ and $\mathrm{Fz}=327.3$ $\pm 19.5 \%$ ). However, the values of the predicted $\mathrm{Fx}$ and $\mathrm{Fz}$ forces using our 
methodology, present a higher variability $(\mathrm{Fx}=200 \pm 61 \%$ and $\mathrm{Fz}=388.7$ $\pm 67.8 \%$ ). This result may indicate that the Fx and Fz force values are highly influenced by the presence of other muscles forces because their values were relatively smaller than the Fy force value. The vertical force (Fy) is considerably higher, and thus, its effect on bone remodelling may be less affected by the presence of other muscle forces and the accuracy of its prediction was so high.

Actually, the knee joint forces are higher than the muscle forces (Marra et al., 2015). In a previous study, Pérez et al. (2010) assumed a physiologicallike loading condition including only the joint reaction force at the condylar surface. They obtained a successful comparison between bone remodeling simulations and CT-data.

Another limitation is the high number of relationships between CT numbers (HU) and bone properties presented in the literature (Pérez et al., 2010). Some of these relationships may not be very accurate and introduce errors in the predictions, although most have proved their effectiveness (Bitsakos et al., 2005). This limitation could be solved if the CT scan is performed using a validated phantom. An accurate estimation of bone density would improve the precision of the subject-specific finite element model (Schileo et al., 2008) and thus the prediction of the subject-specific loads. Additionally, bone properties extracted from the CT scans cannot easily include bone anisotropy, although bone remodelling simulations can take into account this actual anisotropy (Doblaré and García, 2002). For future works, the consideration of more complex image analysis algorithms that allow bone anisotropic properties to be determined will provide additional information 
that would improve the accuracy of the force prediction (Bitsakos et al., 2005; Taghizadeh et al., 2016). When selecting the ROIs regions where we computed the average density values, the upper epiphysis and lower epiphysis and metaphysis were not included because they were very close at the loading and boundary conditions regions. In fact, we performed a sensitive analysis using different and additional ROIs, and we concluded that if we increased the number of ROIs, the results did not improve significantly.

An additional limitation of this methodology was its high computational cost because we needed to run multiple bone remodelling simulations and train every subject-specific ANN to obtain a very accurate result. In a future work, we could use these extrapolation methods to accelerate the bone remodelling simulations (Mohaghegh et al., 2014).

According to Vahdati et al. (2014), the prediction of the density distribution in patients was dependent on the subject-specific loading condition, although they used all the muscles forces. We have clearly demonstrated the relationships between the density values and every subject-specific force (Table 5). Only in certain cases we can interchange the input and subjectspecific ANN (see Table 2, subjects 3 and 4); however, the error predicting the forces increased. Subjects 3 and 4 presented similar characteristics (Table 1) and both were females of similar age. Additionally, the maximum knee joint forces were also very similar (Table 2). Therefore, in certain cases (similar age, gender, or bone density distribution), it would be possible to use a subject-specific ANN previously trained. In a future work, we need to test the methodology in a high sample set to confirm this conclusion.

During the development of the tibia FE models, we made a simplification 
related to cartilage joint between tibia and fibula, assuming a linear and elastic behavior. It is known that articular cartilage behaves nonlinearly and it is not a single-phasic material. The biphasic behavior is important in inststantaneous load application. But the bone remodeling problem has a different timescale, therefore, we could assume cartilage behavior as linear elastic. Additionally, another simplification of our methodology is the use of a phenomenological bone remodeling algorithm (Doblaré and García, 2001), where it is assumed that all bone remodeling is caused by mechanical loading. But hormones and many other biological factors in different individuals can play an important role in bone remodeling. Therefore, a mechanistic bone remodeling algorithm (Huiskes et al., 2000; García-Aznar et al., 2005; Klika et al., 2014) could be used in a future.

The final limitation of the proposed methodology was its small sample set. Four of the five subjects were very young, and the fifth subject was a 61-year-old female, although she had no signs of osteoporosis. In the authors' opinion, however, this limitation does not reduce the importance and generality of the obtained results. As previously indicated, certain subjects could use a previously developed subject-specific ANN-based model. Therefore, a database by trained subject-specific ANNs should be established, which will motivate its clinical application. A database for osteoporotic patients could also be developed using a special mechanistic bone remodeling algorithm (Klika et al., 2014), which will also drive its clinical application.

Despite previous limitations and as fas as author's knowledge, it is the first work in which a quantitative comparison is performed between loads estimated numerically and loads computed by means of gait analysis. There- 
fore, this work also presents a strong scientific impact with relevant clinical implications, because it opens a new way to evaluate patient-specific forces from image-based analysis combined with neural networks, bone remodeling and finite element simulations. Hence, with this new methodology, we have knowledge about the joint loading without the requirement of performing measurements such as done with gait-analyses.

In summary, the methodology presented in this work provides a new strategy for the systematic development of subject-specific load-prediction models, facilitating the application of subject-specific modelling tools for clinical applications.

\section{Acknowledgements}

The authors gratefully acknowledge the support through the CAD-BONE project 286179 of the European Commission and the Spanish Ministry of Economy and Competitiveness through research project DPI 2014-53401C2-1-R.

\section{Appendix}

\section{Appendix A. Bone remodelling model}

The anisotropic bone remodelling model used in this study was based on the principles of continuum damage mechanics (Doblaré and García, 2001). It used a fabric tensor and the apparent density as internal variables to model both the change in bone density distribution and the directionality of the microstructure (Doblaré and García, 2001, 2002; Garijo et al., 2014a). Starting from isotropic material properties, the material will adjust and align 
its principal directions of anisotropy with those of the stress tensor, achieving a directional equilibrium when the stress is aligned with the fabric tensor. The anisotropic bone remodelling algorithm was implemented in an Abaqus UMAT subroutine.

\section{Appendix B. Regions of interest for the tibia}

In the tibial FE model, we selected seven regions of interest (ROIs) to study the average apparent density in these areas (Fig. 5 a-g) (Morgan-Jones et al., 2015). The apparent density $(\rho)$ will come from the bone remodelling simulations or from the subject-specific data (HU) (Fig. 2.1). The diaphysis was divided into four proportional parts (Fig. 5 a-d). In each part, we separated the cortical $\left(\rho \geq 1.2 \mathrm{gr} / \mathrm{cm}^{3}\right)$ and the trabecular bone $(\rho<1.2$ $\mathrm{gr} / \mathrm{cm}^{3}$ ) (Jacobs, 1994) at every integration point of the FE mesh. Therefore, in every region, we computed the average density over the bone tissue volume in that specific region. To summarize, we had eight (Fig. 5 1-8) average density values in these four ROIs. We also divided the proximal metaphysis into two volumes, upper and lower, as two additional inputs (9-10), where we computed their average density (Fig. 5e-f). Therefore, we have ten input data sets that are the average bone density distribution within the ROIs. Additionally, we selected the central area of the diaphysis (Fig. 5g), and we computed the percentage of cortical (11) and trabecular bone volumes (12) as two additional inputs. Therefore, we ultimately had twelve inputs for the ANN (see Appendix C). The upper epiphysis and lower epiphysis and metaphysis were not included because they were very close to the loading and boundary conditions regions. 
[Fig. 5 about here.]

\section{Appendix C. Artificial Neural Network (ANN)}

ANNs are mathematical models inspired by the structure and functional aspects of biological neural networks. A neural network consists of an interconnected group of artificial neurons, and it processes information using a connection approach to computation. The numbers of types of ANNs and their uses are very high. Since the first neural model, proposed by McCulloch and Pitts (1990), other models considered to be ANNs have been developed. The differences among them might be the functions, the accepted values, the topology, the learning algorithms, and so forth. A particular case of a neural network is the multilayer perceptron (MLP) with a layered structure, where each neuron is a perfectron. The functional model of neural networks is based on feedforward networks (MLP) with specific activation functions and weights with fixed values. Algorithms that can adjust the weights of the ANN to obtain the desired output from the network can be found. In this study, the back-propagation algorithm was used for training the ANN, and the function to relate the inputs with the 50 neurons for the hidden layer was a sigmoid-type and linear function for the output layer. More details of this methodology can be found in Garijo et al. (2014b). To evaluate the learning algorithm, we used 10-fold cross validation. Cross validation is a model validation technique for assessing how the results of a statistical analysis are generalized to an independent data set and to avoid dependency on the data used for the training and testing. We used 10-fold cross validation by randomly dividing the data into two segments: one to learn or train the 
model ( $90 \%$ of the data) and another one (10\% of the data) to validate or test the model. This process is performed 10 times (10-fold cross), changing the segments for validation or testing in each process (Garijo et al., 2014b).

\section{Appendix D. Anatomical landmarks of the tibia}

The origin of the local reference frame was defined as the midpoint between the medial (MM) and lateral malleoli (LM) (Fig. 3b). The Y axis was the line connecting the midpoint between the tips of the MM and LM and the midpoint between the most medial point on the border of the medial tibial condyle (MC) and the most lateral point on the border of the lateral tibia condyle (LC). The $\mathrm{Z}$ axis was the line lying in the plane defined by the tips of the MM and LM and the midpoint between the most medial point on the border of the MC and the most lateral point on the border of the $\mathrm{LC}$, perpendicular to the $\mathrm{Y}$ axis, pointing to the right (see Fig. 3b). Finally, the $\mathrm{X}$ axis was the line perpendicular to both the $\mathrm{Y}$ and $\mathrm{Z}$ axes. To localize the knee rotation centre, the positions of the skin markers were identified. The rotation centre of the tibia-fibula joint (RJ) was approximated based on a cylindrical fit through the femoral condyles and the trajectory of the tibiafibula with respect to the femur given by the subject-specific musculoskeletal model (Carbone et al., 2015) (see Fig. 3b).

\section{References \\ References \\ Bitsakos, C., Kerner, J., Fisher, I., Amis, A. A., 2005. The effect of muscle loading on the simulation of bone remodelling in the proximal femur. J.}


Biomech. 38 (1), 133-139.

Bona, M. A., Martin, L. D., Fischer, K. J., 2006. Density-based load estimation using two-dimensional finite element models: a parametric study. Comput. Methods Biomech. Biomed. Engin. 9 (4), 221-229.

Campoli, G., Weinans, H., Zadpoor, A. A., 2012. Computational load estimation of the femur. J. Mech. Behav. Biomed. Mater. 10, 108-119.

Carbone, V., Fluit, R., Pellikaan, P., van der Krogt, M. M., Janssen, D., Damsgaard, M., Vigneron, L., Feilkas, T., Koopman, H. F. J. M., Verdonschot, N., 2015. TLEM 2.0 a comprehensive musculoskeletal geometry dataset for subject-specific modeling of lower extremity. J. Biomech. 48 (5), 734-741.

Carbone, V., van der Krogt, M. M., Koopman, H. F. J. M., Verdonschot, N., 2012. Sensitivity of subject-specific models to errors in musculo-skeletal geometry. J. Biomech. 45 (14), 2476-2480.

Carey, R. E., Zheng, L., Aiyangar, A. K., Harner, C. D., Zhang, X., 2014. Subject-specific finite element modeling of the tibiofemoral joint based on $\mathrm{CT}$, magnetic resonance imaging and dynamic stereo-radiography data in vivo. J. Biomech. Eng. 136 (4), 041004-041004.

Christen, P., van Rietbergen, B., Lambers, F. M., Mueller, R., Ito, K., 2012. Bone morphology allows estimation of loading history in a murine model of bone adaptation. Biomech. Model Mechanobiol. 11 (3-4), 483-492.

Doblaré, M., García, J. M., 2001. Application of an anisotropic boneremodelling model based on a damage-repair theory to the analysis of the 
proximal femur before and after total hip replacement. J. Biomech. 34 (9), $1157-1170$.

Doblaré, M., García, J. M., 2002. Anisotropic bone remodelling model based on a continuum damage-repair theory. J. Biomech. 35 (1), 1-17.

Favre, J., Hayoz, M., Erhart-Hledik, J. C., Andriacchi, T. P., 2012. A neural network model to predict knee adduction moment during walking based on ground reaction force and anthropometric measurements. J. Biomech. 45 (4), 692-698.

Fischer, K. J., Jacobs, C. R., Carter, D. R., 1995. Computational method for determination of bone and joint loads using bone density distributions. J. Biomech. 28 (9), 1127-1135.

Fluit, R., Andersen, M. S., Kolk, S., Verdonschot, N., Koopman, H. F. J. M., 2014. Prediction of ground reaction forces and moments during various activities of daily living. J. Biomech. 47 (10), 2321-2329.

Fluit, R., van der Krogt, M. M., van der Kooij, H., Verdonschot, N., Koopman, H. F. J. M., 2012. A simple controller for the prediction of threedimensional gait. J. Biomech. 45 (15), 2610-2617.

García-Aznar, J., Rueberg, T., Doblaré, M., 2005. A bone remodelling model coupling microdamage growth and repair by 3D BMU-activity. Biomech Model Mechanobiol 4 (2-3), 147-167.

Garijo, N., Fernández, J. R., Pérez, M. A., García-Aznar, J. M., 2014a. Numerical stability and convergence analysis of bone remodeling model. Comput. Methods Appl. Mech. Eng. 271, 253-268. 
Garijo, N., Martínez, J., García-Aznar, J. M., Pérez, M. A., 2014b. Computational evaluation of different numerical tools for the prediction of proximal femur loads from bone morphology. Comput. Methods Appl. Mech. Eng. $268,437-450$.

González-Carbonell, R. A., Ortiz-Prado, A., Jacobo-Armendáriz, V. H., Cisneros-Hidalgo, Y. A., Alpízar-Aguirre, A., 2015. 3D patient-specific model of the tibia from CT for orthopedic use. J. Orthop. 12 (1), 11-16.

Huiskes, R., Ruimerman, R., Van Lenthe, G. H., Janssen, J. D., 2000. Effects of mechanical forces on maintenance and adaptation of form in trabecular bone. Nature 405 (6787), 704-706.

Jacobs, C., 1994. Numerical Simulation of Bone Adaptation to Mechanical Loading. Dissertation for the Degree of Doctor of Philosophy, Stanford University.

Jin, H., Lewis, J. L., 2004. Determination of poissons ratio of articular cartilage by indentation using different-sized indenters. J. Biomech. Eng. $126(2), 138-145$.

Klika, V., Pérez, M. A., García-Aznar, J. M., Maršík, F., Doblaré, M., 2014. A coupled mechano-biochemical model for bone adaptation. J Math Biol 69 (6-7), 1383-1429.

Kolk, S., Minten, M. J. M., van Bon, G. E. A., Rijnen, W. H., Geurts, A. C. H., Verdonschot, N., Weerdesteyn, V., 2014. Gait and gait-related activities of daily living after total hip arthroplasty: A systematic review. Clin. Biomech. 29 (6), 705-718. 
Lekadir, K., Noble, C., Hazrati-Marangalou, J., Hoogendoorn, C., van Rietbergen, B., Taylor, Z. A., Frangi, A., 2015. Patient-specific biomechanical modeling of bone strength using statistically-derived fabric tensors. Annals of Biomedical Engineering .

Liu, Y., Shih, S.-M., Tian, S.-L., Zhong, Y.-J., Li, L., 2009. Lower extremity joint torque predicted by using artificial neural network during vertical jump. J. Biomech. 42 (7), 906-911.

Marra, M. A., Vanheule, V., Fluit, R., Koopman, B. H. F. J. M., Rasmussen, J., Verdonschot, N., Andersen, M. S., 2015. A subject-specific musculoskeletal modeling framework to predict in vivo mechanics of total knee arthroplasty. J. Biomech. Eng. 137 (2), 020904 1-020904 12.

McCulloch, W., Pitts, W., 1990. A logical calculus of the ideas immanent in nervous activity. Bull. Math. Biol. 52 (99-115), 73-97.

Mohaghegh, K., Pérez, M. A., García-Aznar, J. M., 2014. Accelerating numerical simulations of strain-adaptive bone remodeling predictions. Comput. Methods Appl. Mech. Eng. 273, 255-272.

Morgan-Jones, R., Oussedik, S. I. S., Graichen, H., Haddad, F. S., 2015. Zonal fixation in revision total knee arthroplasty. Bone Joint J 97-B (2), $147-149$.

Pellikaan, P., van der Krogt, M. M., Carbone, V., Fluit, R., Vigneron, L. M., Deun, J. V., Verdonschot, N., Koopman, H. F. J. M., 2014. Evaluation of a morphing based method to estimate muscle attachment sites of the lower extremity. J. Biomech. 47 (5), 1144-1150. 
Peng, L., Bai, J., Zeng, X. L., Zhou, Y. X., 2006. Comparison of isotropic and orthotropic material property assignments on femoral finite element models under two loading conditions. Med. Eng. Phys. 28 (3), 227-233.

Pérez, M. A., Fornells, P., Doblaré, M., García-Aznar, J. M., 2010. Comparative analysis of bone remodelling models with respect to computerised tomography-based finite element models of bone. Comput. Methods Biomech. Biomed. Engin. 13 (1), 71-80.

Schileo, E., DallAra, E., Taddei, F., Malandrino, A., Schotkamp, T., Baleani, M., Viceconti, M., 2008. An accurate estimation of bone density improves the accuracy of subject-specific finite element models. J. Biomech. 41 (11), $2483-2491$.

Taghizadeh, E., Reyes, M., Zysset, P., Latypova, A., Terrier, A., Büchler, P., 2016. Biomechanical role of bone anisotropy estimated on clinical ct scans by image registration. Ann. Biomed. Eng. , 1-13.

URL http://dx.doi.org/10.1007/s10439-016-1551-4

Vahdati, A., Walscharts, S., Jonkers, I., García-Aznar, J. M., Sloten, J. V., van Lenthe, G. H., 2014. Role of subject-specific musculoskeletal loading on the prediction of bone density distribution in the proximal femur. J. Mech. Behav. Biomed. Mater. 30 (0), 244-252.

Zadpoor, A. A., Campoli, G., Weinans, H., 2013. Neural network prediction of load from the morphology of trabecular bone. Appl. Math. Model. 37 (7), $5260-5276$. 
Zadpoor, A. A., Weinans, H., 2015. Patient-specific bone modeling and analysis: The role of integration and automation in clinical adoption. J. Biomech. 48 (5), 750-760. 


\section{List of Figures}

1 Schematic flow chart of the steps involved in subject-specific predictions of tibia loading. . . . . . . . . . . . . . . 30

2 Scheme of the comparative and validation analysis of the proposed numerical-based technique and gait analysis. . . . . . . . 31

3 a) FE model and a detail of the proximal condyles where the load is applied. b) Local coordinate frames of tibia and talus segments (MC: most medial point of the medial condyle of the tibia, LC: most lateral point of the lateral condyle of the tibia, MM: medial malleolus, and LM: lateral malleolus) and rotation centre joint (RJ) (see Appendix D). . . . . . . . . . . . . . . . 32

4 Forces Fx, Fy and Fz (N) of the knee joint during the gait cycle. Maximum force values $(N)$ in the $X, Y$, and $Z$ directions (dashed lines) were applied as loading conditions for the tibia bone remodelling simulations. . . . . . . . . . . . . . 33

5 Definition of the input sets for the ANN based on the following ROIs: a-d) diaphysis (4 proportionality parts that separated the cortical and trabecular bone), e) metaphysis (upper), f) metaphysis (lower) and g) central diaphysis (cortical and trabecular bone volume). . . . . . . . . . . . . . . 34 
Fig. 1: Schematic flow chart of the steps involved in subject-specific predictions of tibia loading.

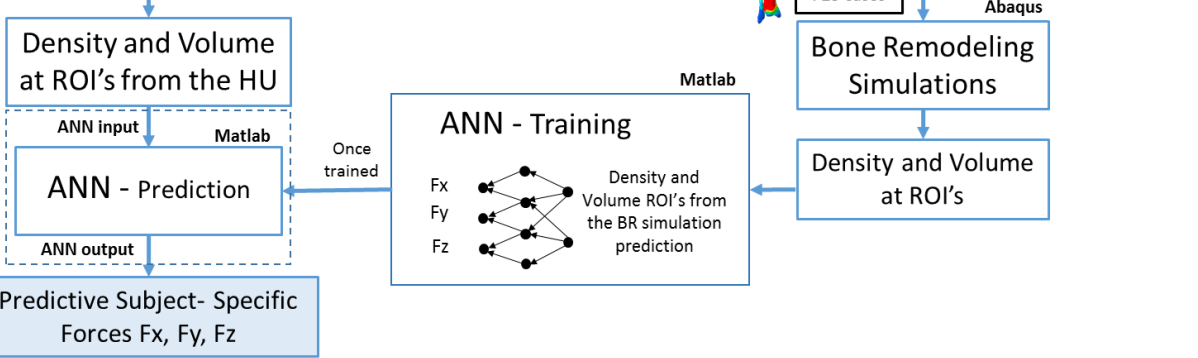


Fig. 2: Scheme of the comparative and validation analysis of the proposed numerical-based technique and gait analysis. 


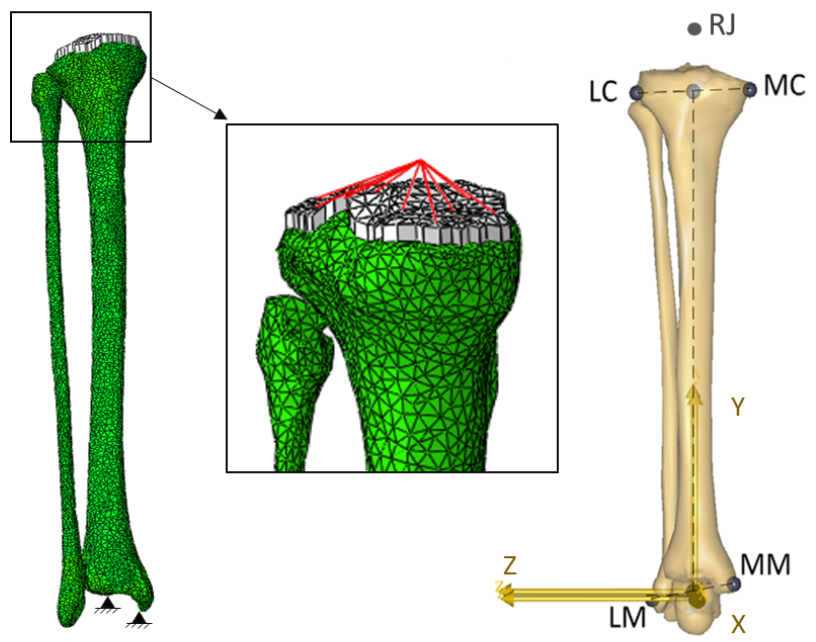

a)

b)

Fig. 3: a) FE model and a detail of the proximal condyles where the load is applied. b) Local coordinate frames of tibia and talus segments (MC: most medial point of the medial condyle of the tibia, LC: most lateral point of the lateral condyle of the tibia, MM: medial malleolus, and LM: lateral malleolus) and rotation centre joint (RJ) (see Appendix D). 


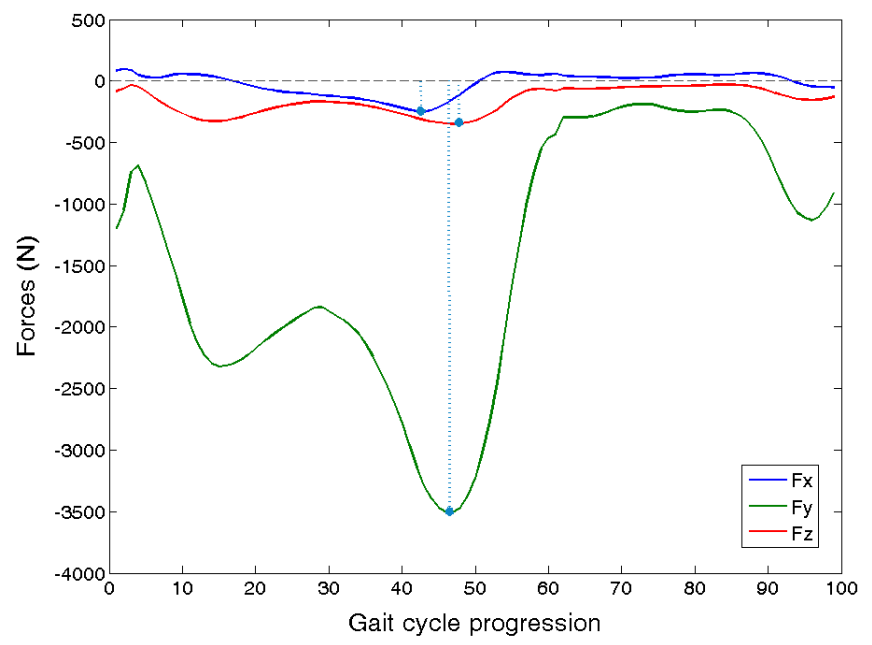

Fig. 4: Forces Fx, Fy and $F z(N)$ of the knee joint during the gait cycles. Maximum force values $(N)$ in the $X, Y$, and $Z$ directions (dashed lines) were applied as loading conditions for the tibia bone remodelling simulations. 
Fig. 5: Definition of the inputs for the ANN based on the following ROIs: a-d) diaphysis (4 proportionality parts that separated the cortical and trabecular bone), e) metaphysis (upper), f) metaphysis (lower) and c) central diaphysis (cortical and trabecular bone volume). 


\section{List of Tables}

1 Subject-specific data used in the study . . . . . . . . . . . 36

2 Maximum knee joint force $(\mathrm{N})$ for each subject from the gait analysis. . . . . . . . . . . . . . . . . . . . . 37

3 Comparison of the relative error (RE) and the correlation coefficient (RSQ) in each subject-specific ANN. . . . . . . . . 38

4 Relative error (RE) \% between forces (N) from gait and ANN predictions for all subjects. . . . . . . . . . . . . . . 39

5 Relative error (RE) \% between forces (N) obtained from gait analysis and those obtained from subject-specific ANN numerical predictions for all subjects. . . . . . . . . . . . . . 40 


\begin{tabular}{|c|c|c|c|c|c|}
\hline Subject & Age & Sex & Weight (Kg) & HU max & $\mathrm{HU} \min$ \\
\hline Subject 1 & 23 & Male & 83.1 & 1603.14 & -72.03 \\
\hline Subject 2 & 26 & Male & 90.4 & 1568.1 & -87.55 \\
\hline Subject 3 & 27 & Female & 58.0 & 1858.8 & -92.21 \\
\hline Subject 4 & 23 & Female & 77.6 & 1579.52 & -65.30 \\
\hline Subject 5 & 61 & Female & 70.7 & 1836.93 & -77.60 \\
\hline
\end{tabular}

Table 1: Subject-specific data used in the study 


\begin{tabular}{lccccccc}
\hline \hline & Subject 1 & Subject 2 & Subject 3 & Subject 4 & Subject 5 & Mean value & Variation (\%) \\
\hline Fx & 156.68 & 153.61 & 117.98 & 126.06 & 156.79 & 137.38 & 14.1 \\
Fy & -2171.38 & -2859.93 & -2013.05 & -1983.66 & -3245.17 & -2614.41 & 24.1 \\
Fz & -391.04 & -318.85 & -369.78 & -314.42 & -263.59 & -327.31 & 19.5 \\
Total force & 2211.87 & 2881.75 & 2050.13 & 2012.38 & 3259.63 & 2636.0 & 23.7 \\
\hline \hline
\end{tabular}

Table 2: Maximum knee joint force $(\mathrm{N})$ for each subject from the gait analysis. 


\begin{tabular}{llccccc}
\hline \hline & & Subject 1 & Subject 2 & Subject 3 & Subject 4 & Subject 5 \\
\hline \multirow{3}{*}{ Force X } & RE Train & $0.082 \%$ & $0.127 \%$ & $0.053 \%$ & $0.04 \%$ & $0.158 \%$ \\
& RE Test & $0.061 \%$ & $0.043 \%$ & $0.019 \%$ & $0.018 \%$ & $0.035 \%$ \\
& RSQ & 0.999 & 0.999 & 0.999 & 0.999 & 0.998 \\
\hline \multirow{3}{*}{ Force Y } & RE Train & $0.006 \%$ & $0.011 \%$ & $0.004 \%$ & $0.002 \%$ & $0.004 \%$ \\
& RE Test & $0.040 \%$ & $0.002 \%$ & $0.001 \%$ & $0.001 \%$ & $0.001 \%$ \\
& RSQ & 0.999 & 0.999 & 0.999 & 0.999 & 0.999 \\
\hline \multirow{3}{*}{ Force Z } & RE Train & $0.060 \%$ & $0.035 \%$ & $0.031 \%$ & $0.020 \%$ & $0.065 \%$ \\
& RE Test & $0.040 \%$ & $0.015 \%$ & $0.010 \%$ & $0.008 \%$ & $0.015 \%$ \\
& RSQ & 0.999 & 0.999 & 0.999 & 0.999 & 0.999 \\
\hline \hline
\end{tabular}

Table 3: Comparison of the relative error (RE) and the correlation coefficient (RSQ) in each subject-specific ANN. 


\begin{tabular}{lccccc}
\hline \hline & & Force X & Force Y & Force Z & Total Force \\
\hline \multirow{3}{*}{ Subject 1 } & Gait & 156.68 & -2171.38 & -391.04 & 2211.87 \\
& ANN & 188.80 & -2014.78 & -493.84 & 2083.00 \\
& Error\% & 20.50 & 7.21 & 26.29 & 5.83 \\
\hline \multirow{3}{*}{ Subject 2 } & Gait & 153.61 & -2859.93 & -318.85 & 2881.75 \\
& ANN & 201.82 & -2780.65 & -443.23 & 2822.97 \\
& Error\% & 31.38 & 2.77 & 39.01 & 2.04 \\
\hline \multirow{3}{*}{ Subject 3 } & Gait & 117.98 & -2013.05 & -369.78 & 2050.13 \\
& ANN & 78.24 & -1934.15 & -652.23 & 2042.66 \\
& Error\% & 33.69 & 3.92 & 76.38 & 0.36 \\
\hline \multirow{3}{*}{ Subject 4 } & Gait & 126.06 & -1983.66 & -314.42 & 2012.38 \\
& ANN & 321.71 & -1905.91 & -125.16 & 1936.92 \\
& Error\% & 155.20 & 3.92 & 60.19 & 3.75 \\
\hline \multirow{3}{*}{ Subject 5 } & Gait & 156.79 & -3245.17 & -263.59 & 3259.63 \\
& ANN & 252.34 & -3198.52 & -283.63 & 3220.97 \\
& Error\% & 60.94 & 1.44 & 7.61 & 1.19 \\
\hline
\end{tabular}

Table 4: Relative error (RE) \% between forces (N) from gait and ANN predictions for all subjects. 


\begin{tabular}{|c|c|c|c|c|c|c|c|c|c|c|c|}
\hline \multicolumn{12}{|c|}{ The absolute relative error (RE)\%. } \\
\hline \multicolumn{2}{|c|}{ Gait Analysis } & \multicolumn{2}{|c|}{ Subject 1 - ANN } & \multicolumn{2}{|c|}{ Subject 2 - ANN } & \multicolumn{2}{|c|}{ Subject 3 - ANN } & \multicolumn{2}{|c|}{ Subject 4 - ANN } & \multicolumn{2}{|c|}{ Subject 5 - ANN } \\
\hline Subject & Force & Force & Error\% & Force & Error\% & Force & Error\% & Force & Error\% & Force & Error\% \\
\hline Subject1 & 2211.87 & 2083.00 & 5.83 & 2752.39 & 24.44 & 2046.59 & 7.47 & 1949.07 & 11.88 & 2628.76 & 18.85 \\
\hline Subject2 & 2881. & 2216.73 & 23.08 & 2822.97 & 2.04 & 2124.59 & 26.27 & 1979.41 & 31.35 & 2549.75 & 11.52 \\
\hline Subject3 & 2050.13 & 2219.48 & 8.26 & 2752.00 & 34.24 & 2042.66 & 0.36 & 2251.94 & 9.84 & 2586.34 & 26.15 \\
\hline Subject 4 & 2012.38 & 2083.50 & 3.53 & 2605.03 & 29.45 & 2024.60 & 0.61 & 1936.92 & 3.75 & 2689.32 & 33.64 \\
\hline Subject5 & 3259.63 & 2640.34 & 19.00 & 3344.09 & 2.59 & 3228.00 & 0.97 & 2865.94 & 12.08 & 3220.97 & 1.19 \\
\hline
\end{tabular}

Table 5: Relative error (RE) \% between forces (N) obtained from gait analysis and those obtained from subject-specific ANN numerical predictions for all subjects. 
LaTeX Source Files
Click here to download LaTeX Source Files: latex.zip

LaTeX Source Files
Click here to download LaTeX Source Files: latex.zip Click here to download LaTex Source Files: latex.zip

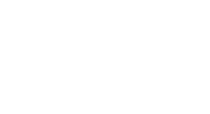
$\sqrt{2}$

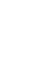

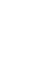

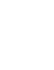

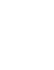
. $\sqrt{3}$ (1) $\sqrt{10}$ (1)

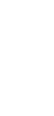

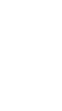
. (2)

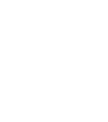
.

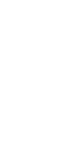

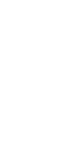
. . . 列 . . . . .

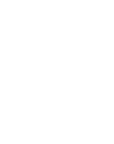

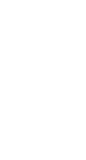

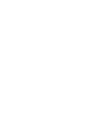

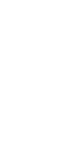

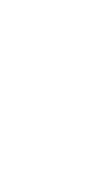

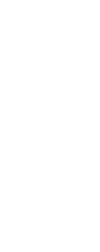
. 更

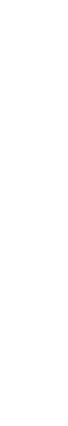

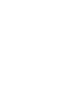

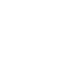

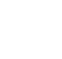

